Research Article

\title{
Determining Pharmacological Mechanisms of Chinese Incompatible Herbs Fuzi and Banxia in Chronic Obstructive Pulmonary Disease: A Systems Pharmacology-Based Study
}

\author{
Kaiwen Ni, ${ }^{1}$ Xiaolu Cai, ${ }^{1}$ Yaling Chen, ${ }^{1}$ Linshui Zhou, ${ }^{2}$ Ruilin Chen, ${ }^{2}$ Suqun Zheng, \\ and Zhen Wang $\mathbb{D}^{1,2}$ \\ ${ }^{1}$ The First Clinical College of Zhejiang Chinese Medical University, Hangzhou, China \\ ${ }^{2}$ The First Affiliated Hospital of Zhejiang Chinese Medical University, Hangzhou, China \\ Correspondence should be addressed to Zhen Wang; wangzhen610@sina.cn
}

Received 18 June 2020; Revised 31 October 2020; Accepted 12 December 2020; Published 31 December 2020

Academic Editor: Akhilesh K. Tamrakar

Copyright (c) 2020 Kaiwen Ni et al. This is an open access article distributed under the Creative Commons Attribution License, which permits unrestricted use, distribution, and reproduction in any medium, provided the original work is properly cited.

\begin{abstract}
Aconiti Lateralis Radix Praeparata (Fuzi) and Pinelliae Rhizoma (Banxia) are among the 18 incompatible medications that are forbidden from use in one formulation. However, there is increasing evidence implying that this prohibition is not entirely correct. According to the theory of Chinese traditional medicine, they can be used for the treatment of chronic obstructive pulmonary disease (COPD). Thus, we analyzed the possible approaches for the treatment of COPD using network pharmacology. The active compounds of Fuzi and Banxia (FB) were collected, and their targets were identified. COPD-related targets were obtained by analyzing the differentially expressed genes between COPD patients and healthy individuals, which were expressed using a Venn diagram of COPD and FB. Protein-protein interaction data and network regarding COPD and drugs used were obtained. Gene ontology and Kyoto Encyclopedia of Genes and Genomes pathway analysis were conducted. The gene-pathway network was constructed to screen the key target genes. In total, 34 active compounds and 47 targets of FB were identified; moreover, 7,153 differentially expressed genes were identified between COPD patients and healthy individuals. The functional annotations of target genes were found to be related to mechanisms such as transcription, cytosol, and protein binding; furthermore, 68 pathways including neuroactive ligand-receptor interaction, Kaposi sarcoma-associated herpesvirus infection, apoptosis, and measles were significantly enriched. FOS CASP3, VEGFA, ESR1, and PTGS2 were the core genes in the gene-pathway network of FB for the treatment of COPD. Our results indicated that the effect of FB against COPD may involve the regulation of immunological function through several specific biological processes and their corresponding pathways. This study demonstrates the application of network pharmacology in evaluating mechanisms of action and molecular targets of herb-opponents FB.
\end{abstract}

\section{Introduction}

As defined by the Global Initiative for Chronic Obstructive Lung Disease (GOLD) Reports 2020, chronic obstructive pulmonary disease (COPD) is characterized by persistent respiratory symptoms and airflow limitation ascribed to airway and/or alveolar abnormalities. Data from the American Lung Association Epidemiology and Statistics Unit show that COPD is projected to be the $3^{\text {rd }}$ by 2020 . According to GOLD, smoking cessation is essential in all COPD patients who continue to smoke, whereas bronchodilators, inhaled corticosteroids (ICS), and anti- inflammatory agents are the primary drugs for COPD patients in stable conditions. However, current treatments are only able to slow the progression of COPD [1].

Recently, traditional Chinese medicine (TCM) has been shown to have potential therapeutic properties against COPD [2]. For instance, Bufei Yishen formula exerted its anti-COPD efficacy by restoring Th17/Treg balance via the modulation of STAT3 and activation of STAT5 [3]. Clinically, chronic and progressive dyspnea are the main characteristic symptoms of COPD, and around a third of patients experience cough and sputum production. According to the theory of TCM, the dyspnea may imply depression of the 
chest yang, while sputum production suggests phlegm stagnant. Additionally, the elderly, the primary population of the disease, are often considered to have declination in the kidney yang. These characteristics mentioned belong to one syndrome called yang-deficiency-with-phlegm-stagnant, in TCM.

In TCM, Aconiti Lateralis Radix Praeparata (Fuzi, the dried lateral root of Aconitum carmichaelii Debx.) improves COPD's yang deficiency by tonifying fire and enhancing yang. Pinelliae Rhizoma (Banxia, the dried tuber of Pinellia ternata (Thunb.) Breit.) defends against COPD's phlegm stagnant by drying dampness and resolving phlegm. In addition, it is reported that $P$. ternata can be used to reverse various adverse effects of the withdrawal of ICS, such as a rebound in goblet cell number, mucin 5AC (MUC5AC) expression, and interleukin $1 \beta$ (IL-1 $\beta$ ) and tumor necrosis factor $\alpha$ (TNF- $\alpha$ ) levels, used for the treatment of COPD [4]. Moreover, patients with COPD have higher levels of systemic inflammation markers [5]. Fuzi has a wide range of biological activities, including anti-inflammatory, antitumor, and immunomodulatory effects, as well as effects on energy metabolism [6].

Cotreatment of FB is not to be used in TCM, as indicated in the Eighteen Incompatible Medicaments Verse and first noted in the Rumen Shiqin [7], owing to high toxicity and side effects of the combination in one apozem. However, increasing evidence shows that they have no significant harmful effects on humans [8]. One clinical study showed that there was no significant toxicity of cotreatment of FB in the heart, liver, kidney, and blood used in patients with malignant tumors. [9] Moreover, as reported in "Shanghan Zabing Lun," a widely used clinical guidebook of TCM, 2000 years ago, FB were used in a formulation called "Fuzi-jingmi Tang." [10] As for now, we still do some clinical researches using formulations which contained FB. Xiaoqinglong-Jiafuzi-Decoction, including both Fuzi and Banxia, significantly reduced COPD patients' modified Medical Research Council (mMRC) scores, and enhanced their lung function. [11] Another clinical research indicated that Xiaoqinglong-Jiafuzi-Decoction sharply increased the negative rate of IgE when compared with conventional Western medicine in the treatment of allergic rhinitis. [12] There was no obvious harmful effectiveness of combination of FB mentioned. Thus, we utilized a network pharmacology method to delineate the mechanisms of action and molecular targets of FB for their possible treatment of COPD.

In TCM, multiple herbs are used in complex herbal formulations for various diseases because of their potentially bioactive components that interact with multiple therapeutic targets. The multicomponent, multitarget, and multipathway of formulations means TCM can provide ideal treatments but also comes with great challenges due to the interactions among them [13]. Network pharmacology is a novel approach that attempts to solve these problems, combining systems network analysis with pharmacology. It can be used to illustrate the synergism among compounds and the potential mechanisms of various components and multitarget drugs at the molecular level through the network levels of compound-compound, compound-target, and target-disease interactions. Network pharmacology could promote the understanding of the interactions among compounds, genes, proteins, and diseases; moreover, it is suitable for the study of complex TCM formulations [14]. For example, a feasible system pharmacology model based on chemical, pharmacokinetic, and pharmacological data was developed via a network construction approach to clarify the synergistic mechanisms of Huangqi (Radix Astragali) and Huanglian (Rhizoma Coptidis) [15]. The active ingredients of Fuzheng Huayu formulation were successfully identified, and the mechanisms by which they inhibit hepatic stellate cell viability were determined using network pharmacology and transcriptomics [16]. In this study, for the first time, we explored the action mechanisms and molecular targets of FB for the treatment of COPD using network pharmacology.

\section{Materials and Methods}

2.1. Active Ingredient Screening. The chemical compositions of FB were identified from the Traditional Chinese Medicine Systems Pharmacology Database and Analysis Platform (TCMSP) [17]. Then, we selected candidate compounds with standard oral bioavailability $(\mathrm{OB}) \geq 30 \%$ and drug-likeness $(\mathrm{DL}) \geq 0.18[18]$.

A total of 34 identified compounds were imported into the DrugBank database [19] to delineate the relevant targets of FB. According to one relevant literature report, 5 representative alkaloids of Fuzi, aconitine (AC), mesaconitine (MA), benzoylaconine (BAC), benzoylmesaconine (BMA), and benzoylhypaconine (BHA), which were not selected out from the DrugBank were also included in the scope of the study [20]. They are also listed in Table 1.

2.2. Identifying COPD-Related Targets. COPD genes were collected from both Genecards and the Online Mendelian Inheritance in Man (OMIM) databases. There was no threshold applied during this step, so as to collect as many genes as possible.

2.3. Venn Diagram. We have made a Venn diagram using Venny to show the overlapping genes of COPD and FB.

2.4. Network and Protein-Protein Interaction Analysis. Using Cytoscape 3.7.2 software, genes common to the drugs and the disease were connected to visualize the relationship between the compounds used against FB and COPD. Protein-protein interactions (PPI), as per data from STRING, are based on the proteins in common. First, the genes are ranked by calculating the number of times they appear in the network. Then, with the CytoNCA APP, we analyzed degree centrality (DC), closeness centrality (CC), local average connectivity-based method (LAC), betweenness centrality (BC), Eigenvector centrality (EC), and network centrality (NC), all of which indicate a protein's topology potential. It is reported that their definitions and calculation formulas have been used in network pharmacology and system 
pharmacology [21]. Meanwhile, we counted the string interactins between nodes to figure out the core genes. To make out the core genes, we used CytoHubba to catch top 5 genes ranked by maximal clique centrality (MCC). [22].

\subsection{Bioinformatic Analysis}

2.5.1. Gene Ontology (GO) Analysis. GO analysis, based on the biological processes, cellular components, and molecular functions, places genes that are functionally similar into one group [23]. We used R and Bioconductor [24] to visualize the GO analysis. Functional classifications were analyzed within genes $(p$ value cutoff $=0.05, q$ value cutoff $=0.05)$.

2.5.2. Kyoto Encyclopedia of Genes and Genomes (KEGG) Database. The KEGG database was used to analyze pathways, in the same manner as GO analysis. Significant changes ( $p$ value cutoff $=0.05, q$ value cutoff $=0.05$ ) were distinguished for further study.

2.6. Visualization of Molecular Docking Analysis of Active Components and Core Genes. The protein expressed by core genes and active components were analyzed to figure out the active ingredients which had both minimum binding energy and polar contact. PYMOL was used to visualize docking effect conformation.

\section{Results}

3.1. Compound-Target Network Analysis. Twenty-one relevant compounds were obtained from the analysis of Fuzi and 13 from Banxia analysis (Table 1). For Fuzi, the OBs of ignavine, (R)-norcoclaurine, and karanjin are $84.08 \%$, $82.54 \%$, and $69.56 \%$, respectively, which suggests that they may be the most effective active compounds present, while for Banxia, the OBs of (3S, 6S)-3-(benzyl)-6-(4-hydroxybenzyl) piperazine-2, 5-quinone, beta-D-ribofuranoside (xanthine-9), and stigmasterol are $46.89 \%, 44.72 \%$, and $43.83 \%$, respectively.

All 39 compounds were imported into the DrugBank database to further delineate the targets of FB. After removing 23 compounds with no known corresponding targets, 6 active compounds in Fuzi and 10 in Banxia were identified. From the active compounds, all 47 targets were identified, 13 for Fuzi (Table S1) and 41 for Banxia (Table S2). Genetic symbols of all targets are included for clarity (Table 1). Additionally, we have listed the number of targets corresponding to different components in FB (Table S3) and included the gene symbols of the targets for clarity. Unfortunately, the 5 compounds that were added were filtered without knowing the corresponding target genes.

3.2. Identifying COPD-Related Targets. COPD genes were collected from both Genecards and the OMIM databases, ultimately resulting in a collection of 7,153 related targets.
3.3. Venn Diagram. A Venn diagram (Figure 1(a)) was used to depict the overlapping genes of COPD and FB. The analysis showed that 44 genes are related to both COPD and FB. Another Venn diagram (Figure 1(b)) was used depict the 7 common genes of FB. The 2 drugs may work synergistically through these common genes: ESR1, PTGS1, PRSS1, PGR, NCOA1, NCOA2, and NR3C2.

3.4. Network Analysis. Using Cytoscape 3.7.2 software, the 44 common genes, alongside the network between FB and COPD, were set up and visualized (Figure 2). Between the 16 compounds in both $\mathrm{FB}$ and the 44 genes with overlapping relationships with $\mathrm{COPD}, 147$ edges were analyzed. These edges indicate the compound-target interactions.

3.5. PPI Analysis. Based on the same 44 targets, after removal of discrete points, a PPI network was generated (Figure 3), which included 39 nodes and 186 edges, representing 39 interacting proteins and 186 interactions. We counted the appearance of genes in the PPI network and ranked their value roughly (Figure 4).

With the use of the CytoNCA APP, we calculated the median of BC, CC, EC, DC, NC, and LAC as 16.4307, 0.5067, $0.1181,8,6.1431$, and 3.75 , respectively. We selected the targets that demonstrated higher indices for each median, for further analysis. Eventually, 13 targets were obtained: FOS, CASP3, VEGFA, ESR1, PTGS2, RELA, AR, CYCS, HIF1A, PGR, PPARG, NCOA1, and NCOA2. In way of CytoHubba, ranked by MCC, we got top 5 genes as core genes- FOS, CASP3, VEGFA, ESR1, and PTGS2 (Table 2 and Figure 5).

3.6. Molecular Docking Analysis. Molecular docking of the active components and core genes with high mutual attraction is listed in Table 3, while the relating images are shown in Figure 6.

3.6.1. GO. The 13 identified candidate targets were analyzed using Bioconducter by $\mathrm{R}$ (threshold; $p$ value cutoff $=0.05, q$ value cutoff $=0.05$ ). The data of GO analysis are listed in Table S4. Twenty GO terms were analyzed (Figure 7).

3.6.2. KEGG. The critical pathways involved in the FB treatment of COPD were identified by KEGG pathway analysis (Figure 8). The data of KEGG analysis are shown in Table S5. There were 68 enhanced pathways identified ( $p$ value cutoff $=0.05, q$ value cutoff $=0.05$ ), including neuroactive ligand-receptor interaction, human immunodeficiency virus 1 infection, Kaposi sarcoma-associated herpesvirus infection, cholinergic synapse, and hepatitis B. Figure 9 shows the details of four pathways through which the genes were primarily distributed in the p53 signaling pathway, apoptosis pathway, and cholinergic synapse pathway.

The article flow chart to show our job is given in Figure 10. 
TABle 1: Components of Fuzi and Banxia and their parameters.

\begin{tabular}{|c|c|c|c|c|}
\hline Id & Name & $\mathrm{OB}$ & $\mathrm{DL}$ & Source \\
\hline MOL002421 & Ignavine & 84.08 & 0.25 & Fuzi \\
\hline MOL002419 & (R)-norcoclaurine & 82.54 & 0.21 & Fuzi \\
\hline MOL002398 & karanjin & 69.56 & 0.34 & Fuzi \\
\hline MOL002388 & Delphin_qt & 57.76 & 0.28 & Fuzi \\
\hline MOL002395 & Deoxyandrographolide & 56.3 & 0.31 & Fuzi \\
\hline MOL002415 & 6-Demethyldesoline & 51.87 & 0.66 & Fuzi \\
\hline MOL002397 & Karakoline & 51.73 & 0.73 & Fuzi \\
\hline MOL002422 & Isotalatizidine & 50.82 & 0.73 & Fuzi \\
\hline MOL002392 & Deltoin & 46.69 & 0.37 & Fuzi \\
\hline MOL002401 & Neokadsuranic acid B & 43.1 & 0.85 & Fuzi \\
\hline MOL002433 & $\begin{array}{l}\text { (3R, 8S, 9R, 10R, 13R, 14S, 17R)-3-hydroxy-4, 4, 9, 13, 14-pentamethyl-17-[(E, 2R)-6-methyl-7-[(2R, } \\
\text { 3R, 4S, 5S, 6R)-3, 4, 5-trihydroxy-6-[[(2R, 3R, 4S, 5S, 6R)-3, 4, 5-trihydroxy-6-(hydroxymethyl)oxan-2- } \\
\text { yl]oxymethyl] oxan-2-yl] oxyhept-5-en-2-yl]-1, 2, 3, 7, 8, 10, 12, 15, 16, 17-decahydr }\end{array}$ & 41.52 & 0.22 & Fuzi \\
\hline MOL002211 & 11,14 -Eicosadienoic acid & 39.99 & 0.2 & Fuzi \\
\hline MOL002406 & 2, 7-Dideacetyl-2, 7-dibenzoyl-taxayunnanine F & 39.43 & 0.38 & Fuzi \\
\hline MOL002434 & Carnosifloside I_qt & 38.16 & 0.8 & Fuzi \\
\hline MOL000359 & Sitosterol & 36.91 & 0.75 & Fuzi \\
\hline MOL002393 & Demethyldelavaine A & 34.52 & 0.18 & Fuzi \\
\hline MOL002394 & Demethyldelavaine B & 34.52 & 0.18 & Fuzi \\
\hline MOL002410 & Benzoylnapelline & 34.06 & 0.53 & Fuzi \\
\hline MOL002423 & Jesaconitine & 33.41 & 0.19 & Fuzi \\
\hline MOL000538 & Hypaconitine & 31.39 & 0.26 & Fuzi \\
\hline MOL002416 & Deoxyaconitine & 30.96 & 0.24 & Fuzi \\
\hline MOL002089 & Mesaconitine & 8.7 & 0.25 & Fuzi \\
\hline MOL002424 & Aconitine & 7.87 & 0.23 & Fuzi \\
\hline MOL002408 & Benzoylaconine & 12.83 & 0.25 & Fuzi \\
\hline MOL002409 & Benzoylhypaconine & 8.7 & 0.29 & Fuzi \\
\hline MOL002093 & Benzoylmesaconine & 8.55 & 0.27 & Fuzi \\
\hline MOL006957 & (3S, 6S)-3-(benzyl)-6-(4-hydroxybenzyl) piperazine-2, 5-quinone & 46.89 & 0.27 & Banxia \\
\hline MOL006967 & beta-D-ribofuranoside, xanthine- 9 & 44.72 & 0.21 & Banxia \\
\hline MOL000449 & Stigmasterol & 43.83 & 0.76 & Banxia \\
\hline MOL006937 & 12, 13-Epoxy-9-hydroxynonadeca-7, 10-dienoic acid & 42.15 & 0.24 & Banxia \\
\hline MOL002776 & Baicalin & 40.12 & 0.75 & Banxia \\
\hline MOL006936 & 10, 13-Eicosadienoic & 39.99 & 0.2 & Banxia \\
\hline MOL003578 & Cycloartenol & 38.69 & 0.78 & Banxia \\
\hline MOL000358 & Beta-sitosterol & 36.91 & 0.75 & Banxia \\
\hline MOL001755 & 24-Ethylcholest-4-en-3-one & 36.08 & 0.76 & Banxia \\
\hline MOL002670 & Cavidine & 35.64 & 0.81 & Banxia \\
\hline MOL002714 & Baicalein & 33.52 & 0.21 & Banxia \\
\hline MOL000519 & Coniferin & 31.11 & 0.32 & Banxia \\
\hline MOL005030 & Gondoic acid & 30.7 & 0.2 & Banxia \\
\hline
\end{tabular}

\section{Discussion}

Network-based methods are expected to make drug discovery breakthroughs by increasing our understanding of drug actions using multiple layers of information. Moreover, it provides additional support in the development of drug design and determination of the mechanism of action [25]. It is also a useful tool to elucidate the potential mechanisms of action of Chinese herbs on diseases, which may determine whether some theories of TCM are correct or incorrect.

As mentioned above, FB may treat COPD; thus, we evaluated this possibility using network pharmacology. Previously, FB were thought to be incompatible based on the commonsense logic employed in TCM. However, so far, no significant and definite adverse effects have been reported by administering a combination of FB. Many studies have focused on the side effects (hepatorenal toxicity) of FB, and a few articles focus on the therapeutic effect of them. We think that it is better to discuss the side effects of drugs in the context of sufficient clinical efficacy. Drugs possess therapeutic effects and may also cause complications; for example, tacrolimus (FK506) and other immunosuppressants inhibit the immunity of patients undergoing transplantation, making them vulnerable to infectious diseases (e.g., BK virus infection) or hypertension mentioned in the drug specification. However, owing to their therapeutic effect, their risks are tolerated. Therefore, it is necessary to study and overcome the possible side effects of FB; however, proving the drugs' therapeutic effect seems to have more clinical value.

In this study, we evaluated some valuable compounds, such as baicalein in Banxia and ignavine and karanjin in Fuzi. Ignavine is a novel allosteric modulator of the $\mu$ opioid receptor and has an analgesic effect in vivo [26]. Karanjin decreases reactive oxygen species (ROS) levels 


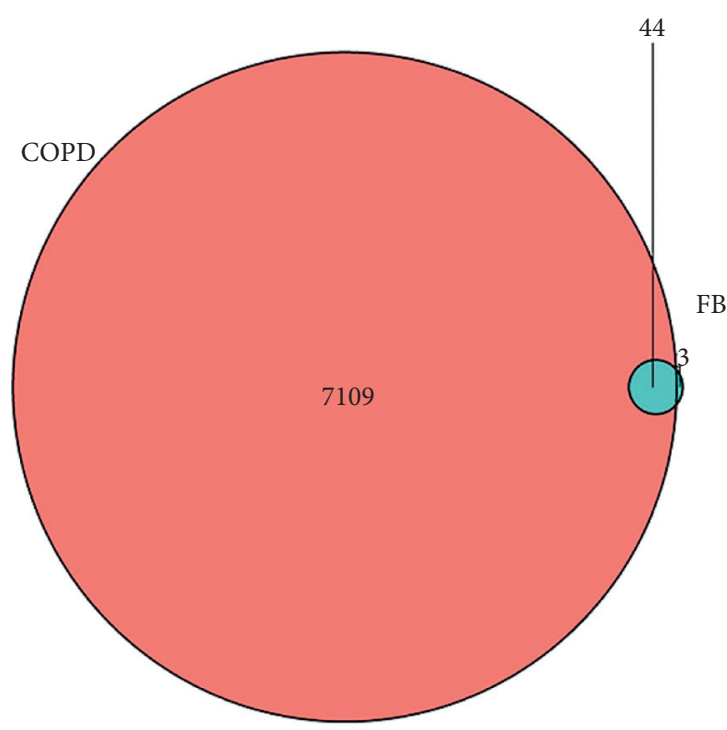

(a)

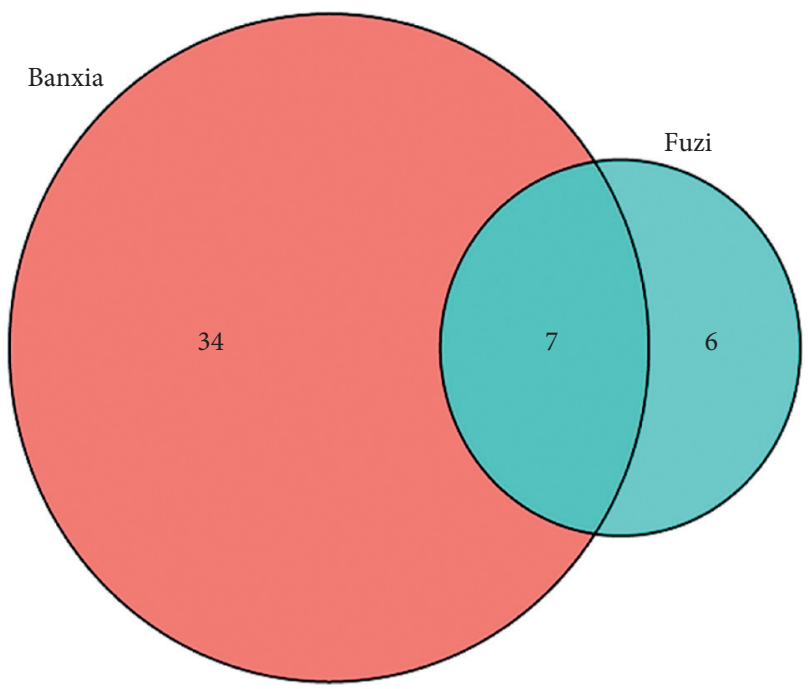

(b)

Figure 1: (a) Venn diagram of overlapping genes related to COPD and FB. Blue indicates genes related to FB, and pink indicates genes related to COPD. (b) Venn diagram of overlapping genes related to Fuzi and Banxia. Blue indicates genes related to Fuzi, and pink indicates genes related to Banxia.

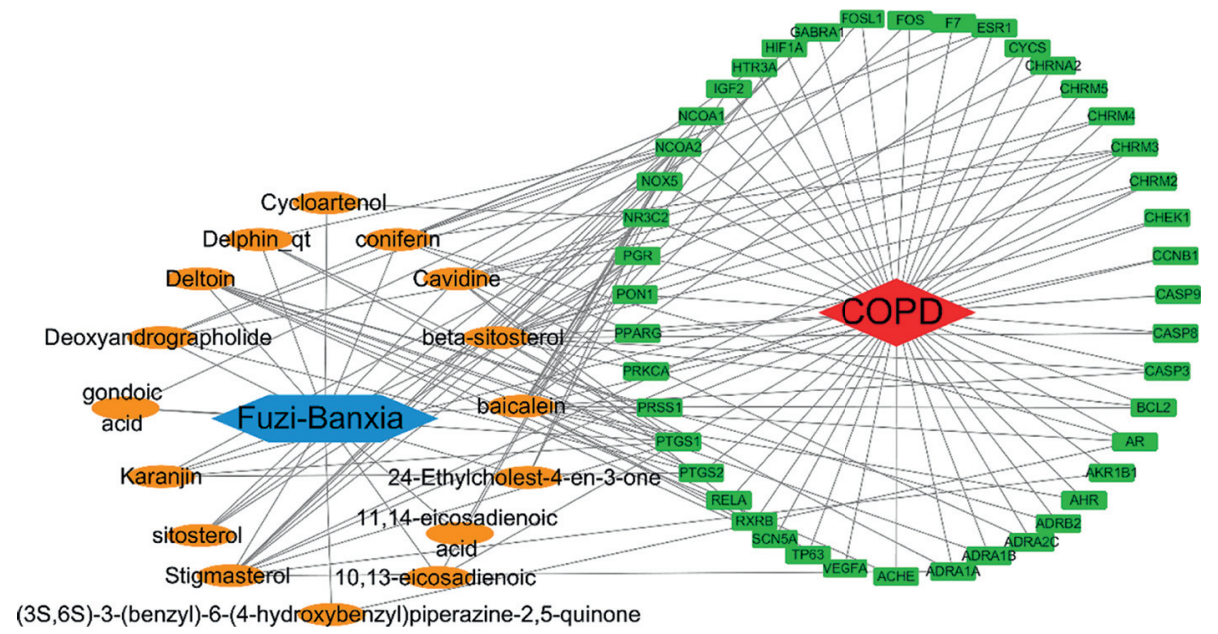

Figure 2: Network analysis of common targets for COPD and FB. Red represents COPD, green represents relevant genes, orange represents the ingredients, and blue represents the impairing drugs.

by inhibiting B-cells inhibitor $(\mathrm{I}-\kappa \mathrm{B})$, resulting in the restriction of nuclear factor kappa-light-chain-enhancer of activated B-cells (NF- $\kappa \mathrm{B})$ nuclear translocation. It can also reduce DNA damage by increasing p53 expression [27]. Karanjin has been shown to reduce TNF- $\alpha$ production and to have a potent inhibitory effect on nitric oxide and reactive oxygen species production [28]. As oxidative stress is a major contributor to the pathogenesis of COPD, karanjin may be effective in the treatment of COPD. Although AC, HA, MA, BAC, BHA, and BMA were filtered out, they are the main research targets in experiments related to Fuzi. A study based on ultra-highperformance liquid chromatography coupled tandem mass spectrometry (UPLC-MS/MS) showed that the parent compounds (AC, HA, and MA), which are more toxic than their corresponding secondary metabolites (BAC, BHA, and BMA), would be eliminated more rapidly [29]. In turn, the components found in this study, which are not regarded as the key active components of Fuzi, may have new and significant properties, which may be potentially demonstrated in subsequent studies. Baicalein, from Banxia, has been shown to relax rat tracheal smooth muscle, as effectively as theophylline [30].

This study demonstrates that there are 44 overlapping genes between FB and COPD (Figure 1). The PPI network analysis of FB's putative targets and COPD-related targets 


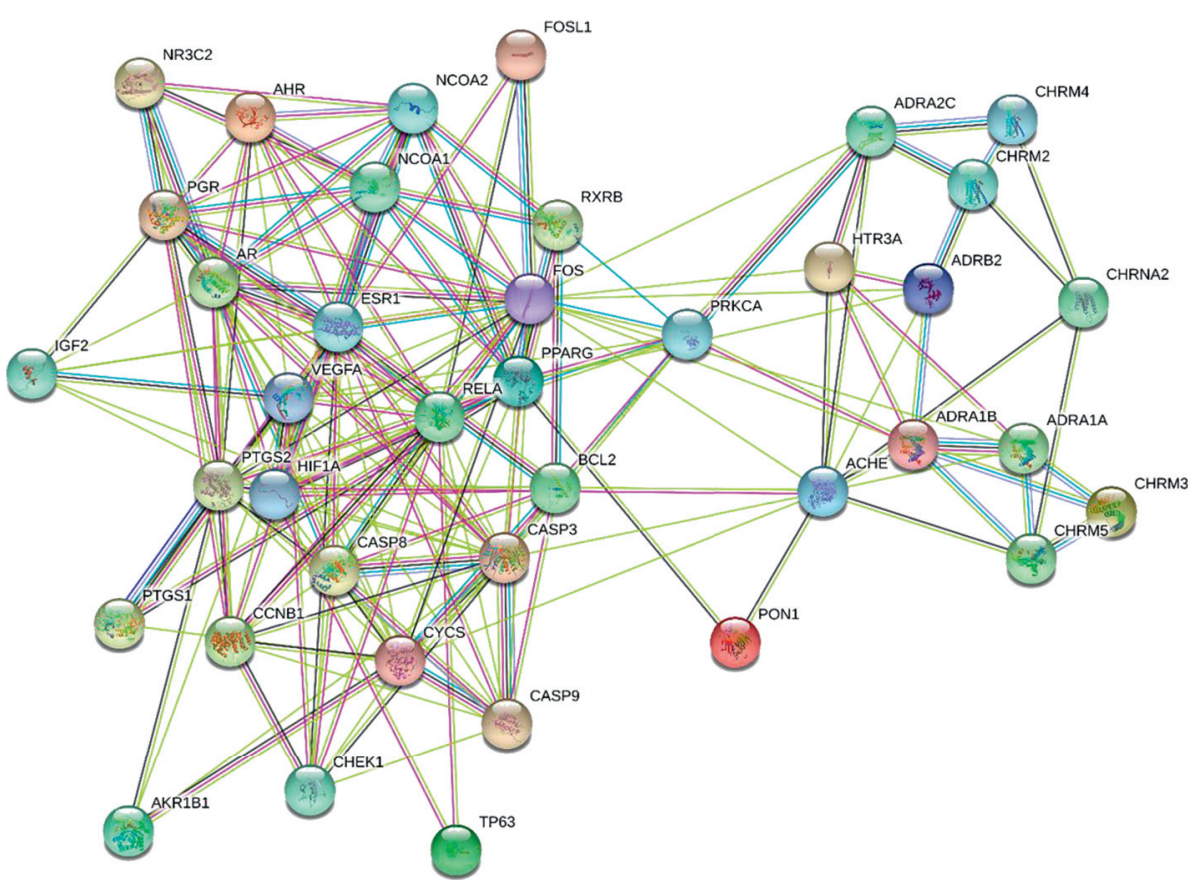

FIgURE 3: PPI network for COPD and FB.

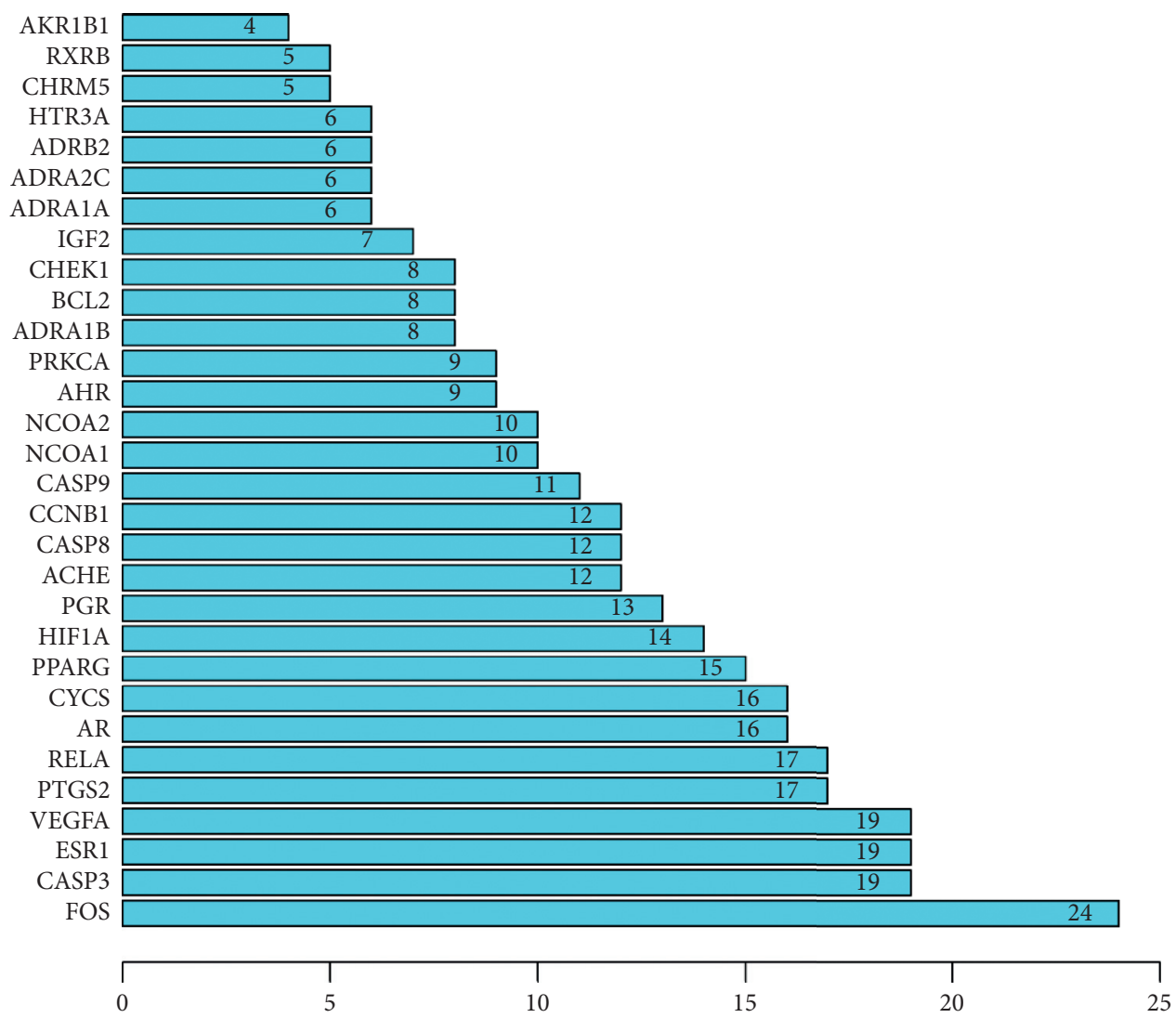

FIgUre 4: Bar plot of PPI for COPD and FB. The number showed the appearance times of the genes in PPI. Larger number means more important. 
TABLe 2: Targets identified above each median.

\begin{tabular}{|c|c|c|c|c|c|c|}
\hline & Degree & Eigenvector & LAC & Betweenness & Closeness & Network \\
\hline FOS & 24 & 0.30 & 8.58 & 379.38 & 0.73 & 19.12 \\
\hline CASP3 & 19 & 0.28 & 9.68 & 75.48 & 0.63 & 15.54 \\
\hline VEGFA & 19 & 0.28 & 9.68 & 56.57 & 0.61 & 16.07 \\
\hline ESR1 & 19 & 0.28 & 9.79 & 52.61 & 0.60 & 16.30 \\
\hline PTGS2 & 17 & 0.26 & 9.18 & 65.09 & 0.61 & 13.13 \\
\hline RELA & 17 & 0.25 & 8.47 & 70.93 & 0.59 & 13.15 \\
\hline $\mathrm{AR}$ & 16 & 0.25 & 9.25 & 38.73 & 0.58 & 13.01 \\
\hline CYCS & 16 & 0.24 & 8.25 & 62.59 & 0.60 & 12.18 \\
\hline HIF1A & 14 & 0.22 & 7.29 & 45.81 & 0.57 & 9.76 \\
\hline PGR & 13 & 0.20 & 7.69 & 26.13 & 0.55 & 10.18 \\
\hline PPARG & 15 & 0.19 & 5.47 & 108.42 & 0.58 & 8.28 \\
\hline NCOA1 & 10 & 0.14 & 5.40 & 22.35 & 0.53 & 7.01 \\
\hline NCOA2 & 10 & 0.14 & 5.40 & 22.35 & 0.53 & 7.01 \\
\hline
\end{tabular}

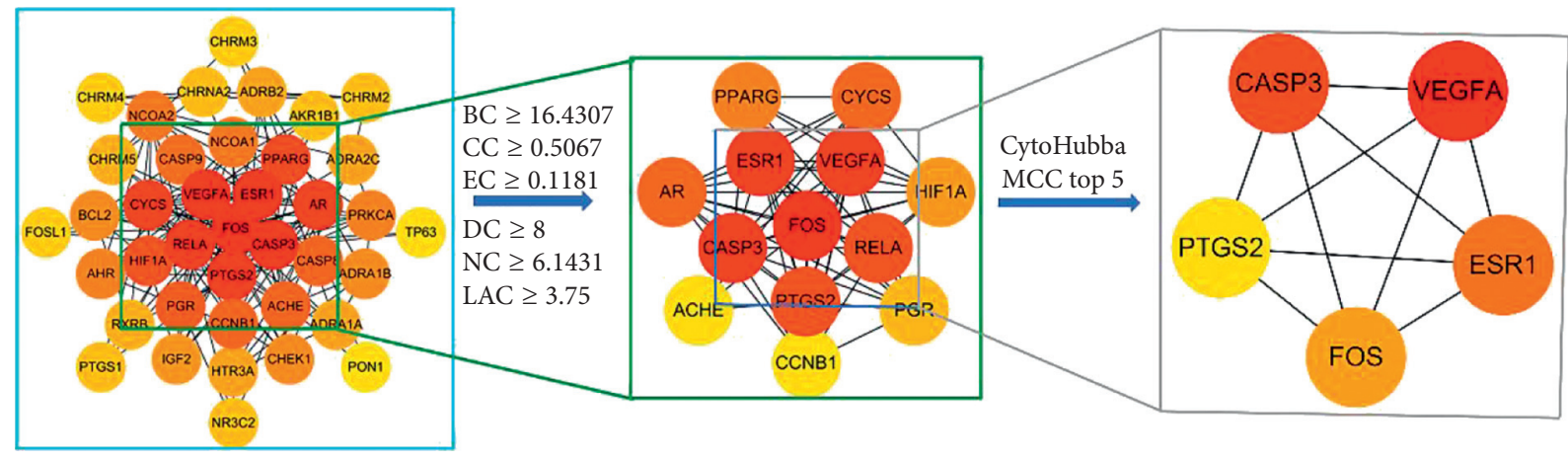

(a)

(b)

(c)

Figure 5: (a). Identification of targets of FB against COPD. From red to yellow indicates higher DC scores to low DC scores. (b) Targets identified above the medians. From red to yellow indicates higher DC scores to lower DC scores. (c). Core genes of FB and COPD.

TABLE 3: Molecular docking of core genes and active components.

\begin{tabular}{lcc}
\hline Core gene & Active component & Binding energy \\
\hline Caspase-3 & Stigmasterol & -5.35 \\
ESR1 & Stigmasterol & -5.41 \\
FOS & Cavidine & -5.38 \\
PTGS2 & Stigmasterol & -4.01 \\
VEGFA & Cavidine & -4.07 \\
\hline
\end{tabular}

was explored and identified common targets that scored higher than the medians in the analyzed parameters. The top 13 targets identified were FOS, CASP3, VEGFA, ESR1, PTGS2, RELA, AR, CYCS, HIF1A, PGR, PPARG, NCOA1, and NCOA2.

Oxidative stress can destroy biomacromolecules in tissues, leading to cell dysfunction or cell death [31]. This study shows that the correlated target genes or pathways are highly associated with oxidative stress, which is a major contributor to the pathogenesis of COPD. For example, Recuperating Lung Decoction could inhibit the MAPK/AP-1 signaling pathway to downregulate oxidative stress, resulting in the improvement of antioxidation of COPD. [32] Indeed, apoptosis was the second most indicated pathway in KEGG analysis. Whether in p53 signaling way, Kaposi sarcomaassociated herpesvirus infection pathway, or human immunodeficiency virus 1 infection pathway, the genes were primarily involved in apoptosis pathway (Figure 10). CSE induced endothelial apoptosis in COPD through the ERK pathway [33]. In turn, oxidative stress can promote the development of lung inflammation and form a chronic state of mucus secretion. The Fos-related antigen-1 (Fra-1) transcription factor is thought to play a key role in promoting chronic cigarette smoke- (CS-) induced lung macrophagic inflammation in vivo and experiments have shown that the absence of Fra-1 in mice bone marrow can reduce the expression of inflammatory factors and the aggregation of macrophages in the lung [34]. Additionally, Fos-related antigen-2 (Fra-2) expression has also been described in COPD for the upregulation of monocyte-derived macrophages upon CS stimulation [35]. Activating transcription factor 3 (ATF3) may be involved in transcriptional promotion of CS-induced MUC5AC expression, which is a key pathologic feature of COPD in airway epithelial cells. Moreover, the knockout of ATF3 can significantly reduce the production of mucus and reduce the chronic mucus hypersecretion of airways [36].

Protease-antiprotease imbalance is the third main pathogenic mechanism of COPD. CS leads to the infiltration of many neutrophils and macrophages in the lung, releasing excessive neutrophil elastase and matrix metalloproteinase 


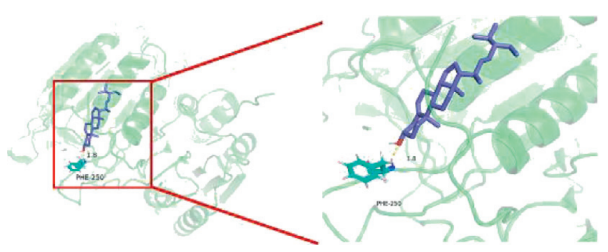

(a)

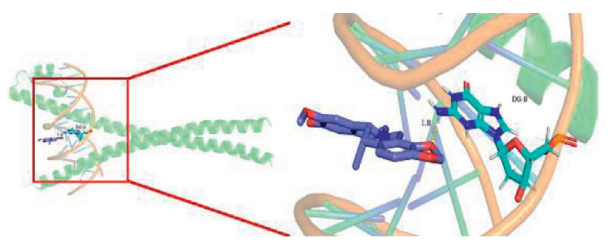

(c)

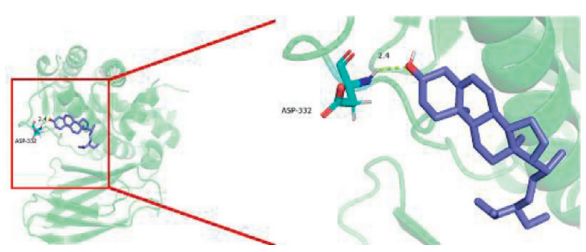

(b)

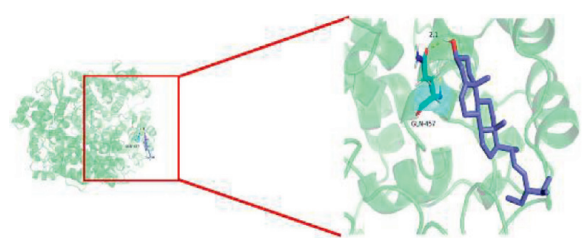

(d)

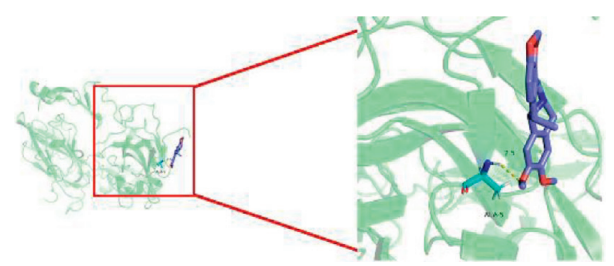

(e)

FIGURE 6: Molecular docking charts of core genes and active components. (a) CASP3 and Stigmasterol. (b) ESR1 and Stigmasterol. (c) FOS and Cavidine. (d) PTGS2 and Stigmasterol. (e) VEGFA and Cavidine.

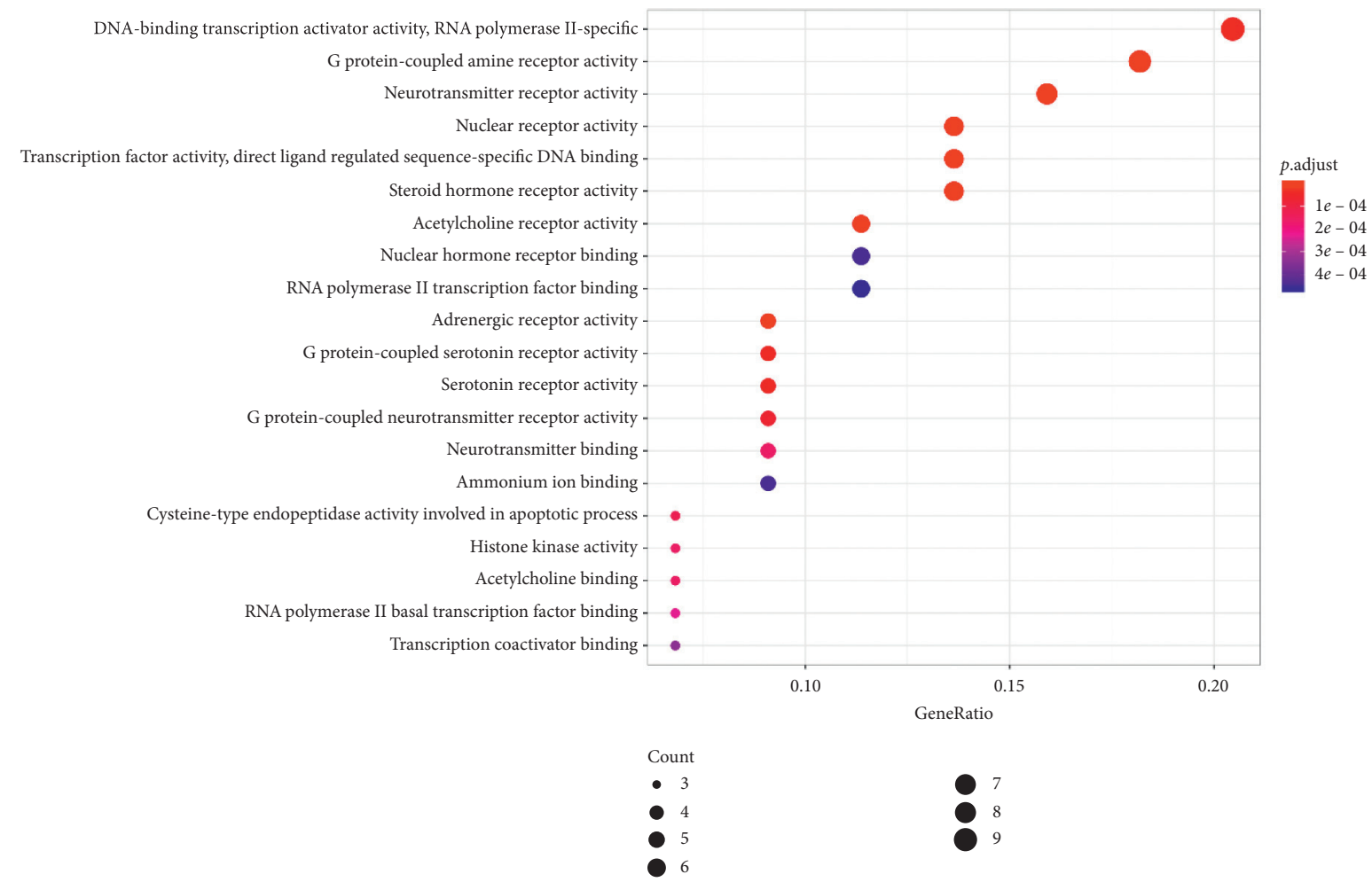

FIgURE 7: Gene ontology terms of candidate targets of FB against COPD. They were identified by the threshold of $p$ value cutoff $\leq 0.05$. The size of the spot represents the number of genes, and the color represents the adjusted $p$ value.

(MMP). Through the protease antiprotein imbalance mechanism, the expression of placenta growth factor (PGF) is increased and drives caspase-3- and caspase-9-dependent apoptosis in bronchial epithelial cells [37]. Pulmonary emphysema, a significant characteristic of COPD, is considered to result from the epithelial cell death caused by 


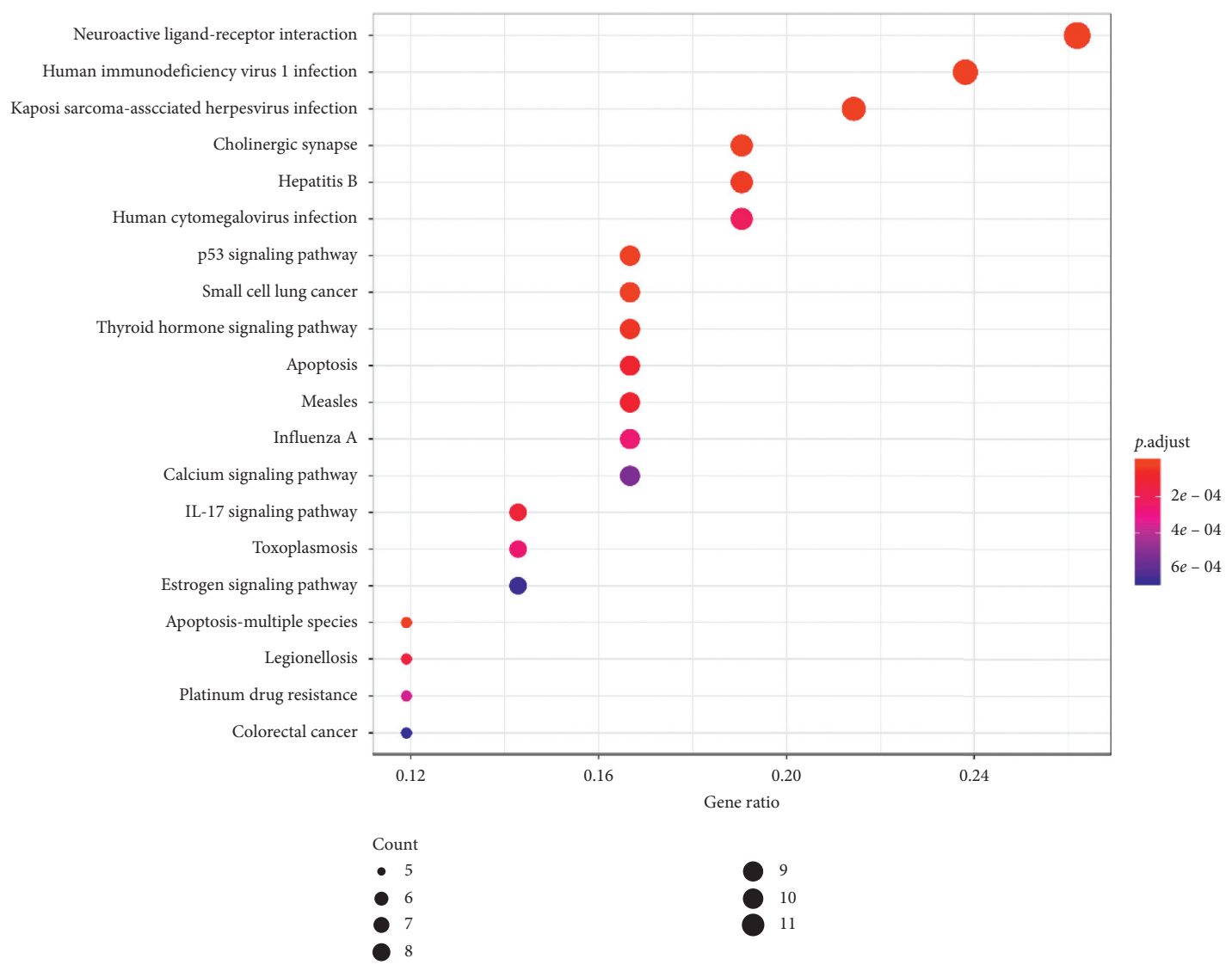

FIGURE 8: KEGG pathway identification of FB candidate targets against COPD. They were identified by the threshold of value cutoff $\leq 0.05$. The size of the spot represents the number of genes, and the color represents the adjusted value.

smoking [38]. It shows that the normal pulling force of alveoli to small airways has been reduced, making the alveoli easily collapsible with obviously reduced elasticity. The failure of the small airway is an important but easily overlooked mechanism of COPD because it occurs in the very early stages of the disease, and continues throughout the process [39]. Small airway disease is composed of small airway inflammation, fibrotic tissue, and lumen mucus plug. The the abovementioned mechanisms jointly promote the most characteristic symptom of COPD: continuous airflow restriction. ICS are very effective in the treatment of COPD. However, ICS resistance is becoming a major barrier to the effective treatment of COPD [40]. There has been evidence indicating that the loss of Hsp90 could contribute to steroid resistance in COPD [41]. Meanwhile, one clinical study has illustrated that smokers with COPD possessed significantly increased expression levels of p53 when compared to smokers without COPD and normal subjects, and at the same time, increased cleaved caspase- 3 may also promote apoptosis [42]. All these biological processes were analyzed in the present study. As shown in Figure 8, the caspase-3, caspase-9, and p53 signaling pathways form the core of the network between FB and COPD. Moreover, the highlighted parts in the major pathways are mainly related to apoptosis.

As is demonstrated in the present study, $\mathrm{FB}$ are multicomponent, multitarget, and multipathway drugs. A total of 68 pathways were enhanced in this drug, including those related to IL-17 signaling, P53 signaling, cholinergic synapse, and apoptosis. IL-17A regulates airway inflammation, oxidative stress, and the reduction of steroid sensitivity [43], as well as attenuating IFN- $\lambda$ expression [44] in COPD. Cellular senescence and apoptosis of alveolar epithelial cells in lung tissues are important characteristics of COPD pathogenesis. p53 is a tumor suppressor, which can induce cellular senescence of type II alveolar epithelial cells (AECII) [45]. p53 expression in the cytoplasm also increased with CSE treatment. In addition, the interaction of p53 with Parkin (a core mitophagy-regulating protein) was highly increased during CSE-induced cellular senescence [46]. The cholinergic anti-inflammatory pathway was involved in regulating inflammation; moreover, COPD mouse showed high levels of AChE and nicotinic acetylcholine receptor $\alpha 7$ subunit ( $\alpha 7 \mathrm{nAChR}$ ) [47]. The nonneuronal cholinergic system was overexpressed in neutrophils of COPD patients, such as the increase of muscarinic receptors (M2, M4, and M5) expression [48]. Additionally, in this study, several other viral pathways were enhanced. Studies which use polymerase chain reaction have demonstrated the presence of viruses in $80-85 \%$ of asthma exacerbations in children and $60-80 \%$ of exacerbations in adults, after viral clearance, and symptom resolution follows, but lasting inflammatory markers and oxidative 


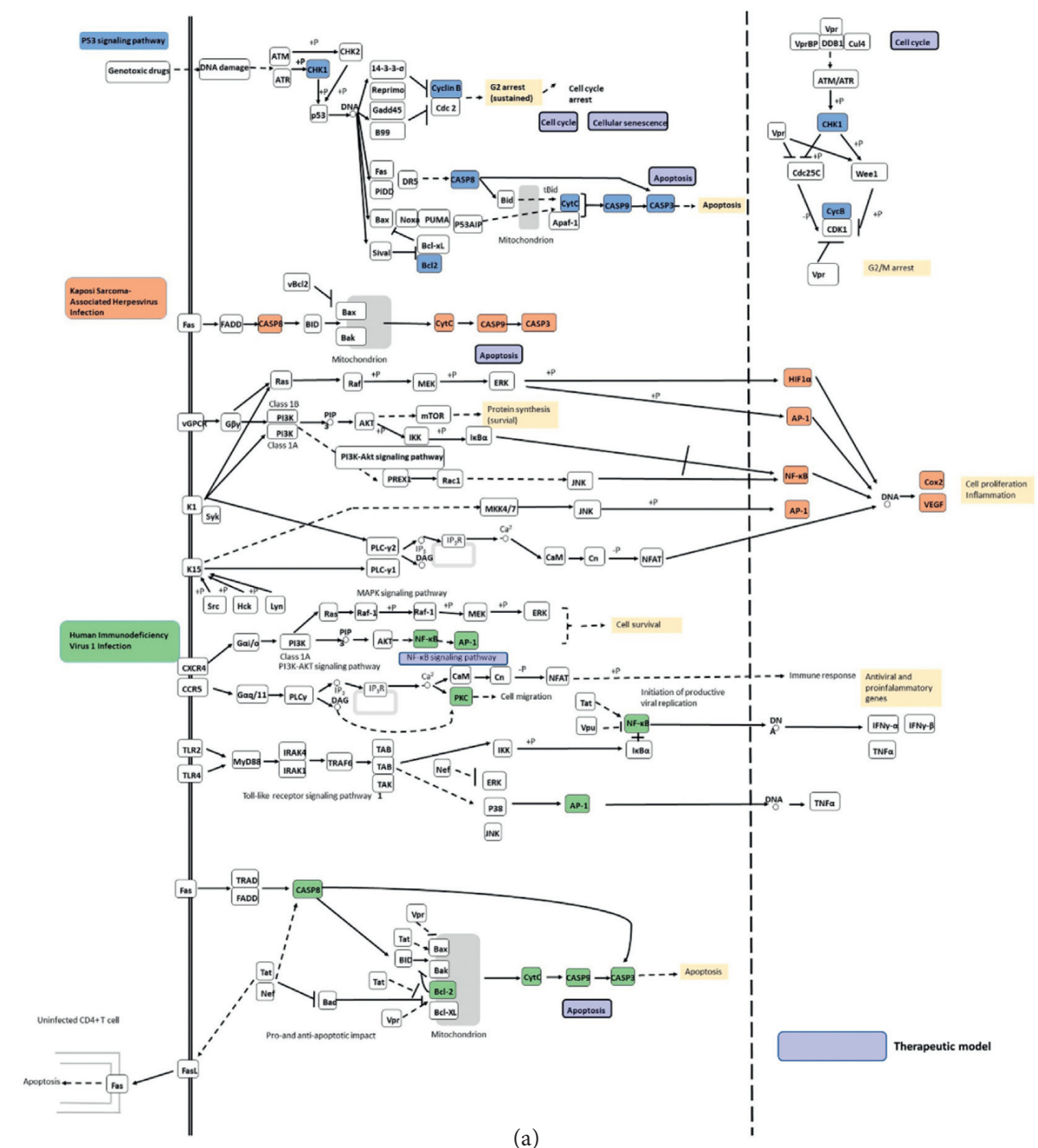

(a)

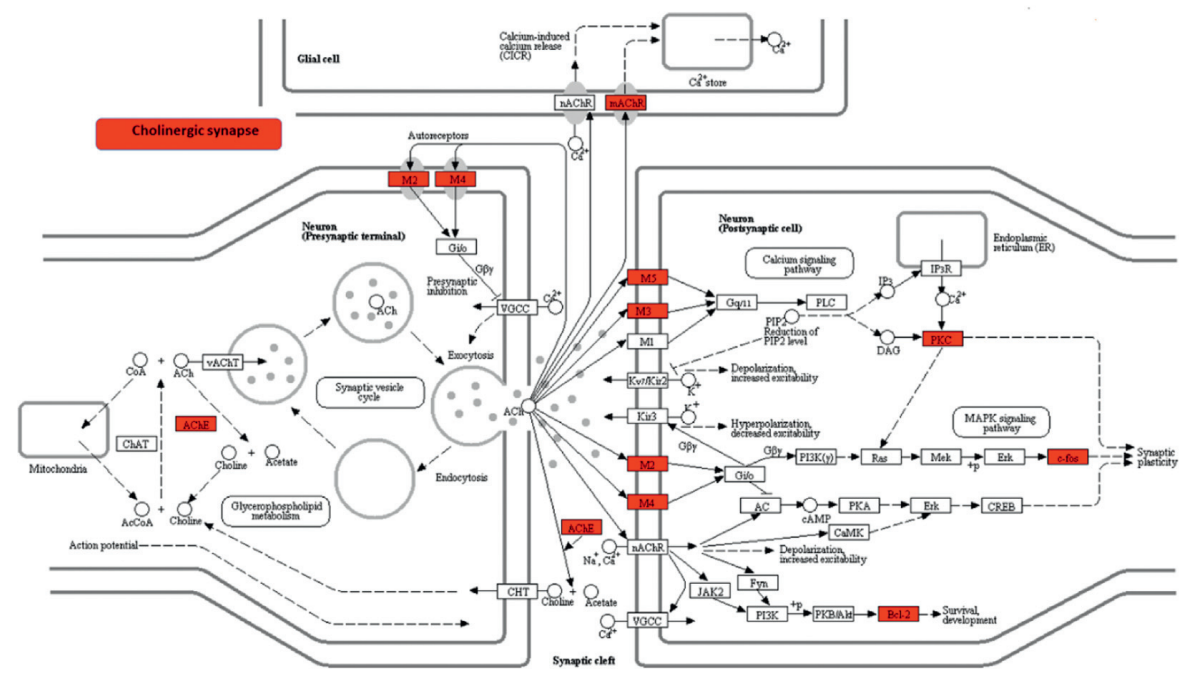

(b)

Figure 9: (a) KEGG pathway details identified by FB against COPD. Three pathways (shown in different colors) consisted the compressed KEGG pathway. The solid and dashed arrows mean direct and indirect activations, and the T arrows indicate the inhibition effects. (b) Cholinergic synapse details identified by FB against COPD.

stress remain [49], which suggests a perpetual autoimmune response. FB may regulate the immunological function through some specific infections' pathways, such as Kaposi sarcoma-associated herpesvirus infection, human immunodeficiency virus 1 infection, human cytomegalovirus infection, hepatitis $C$, influenza $A$, and 


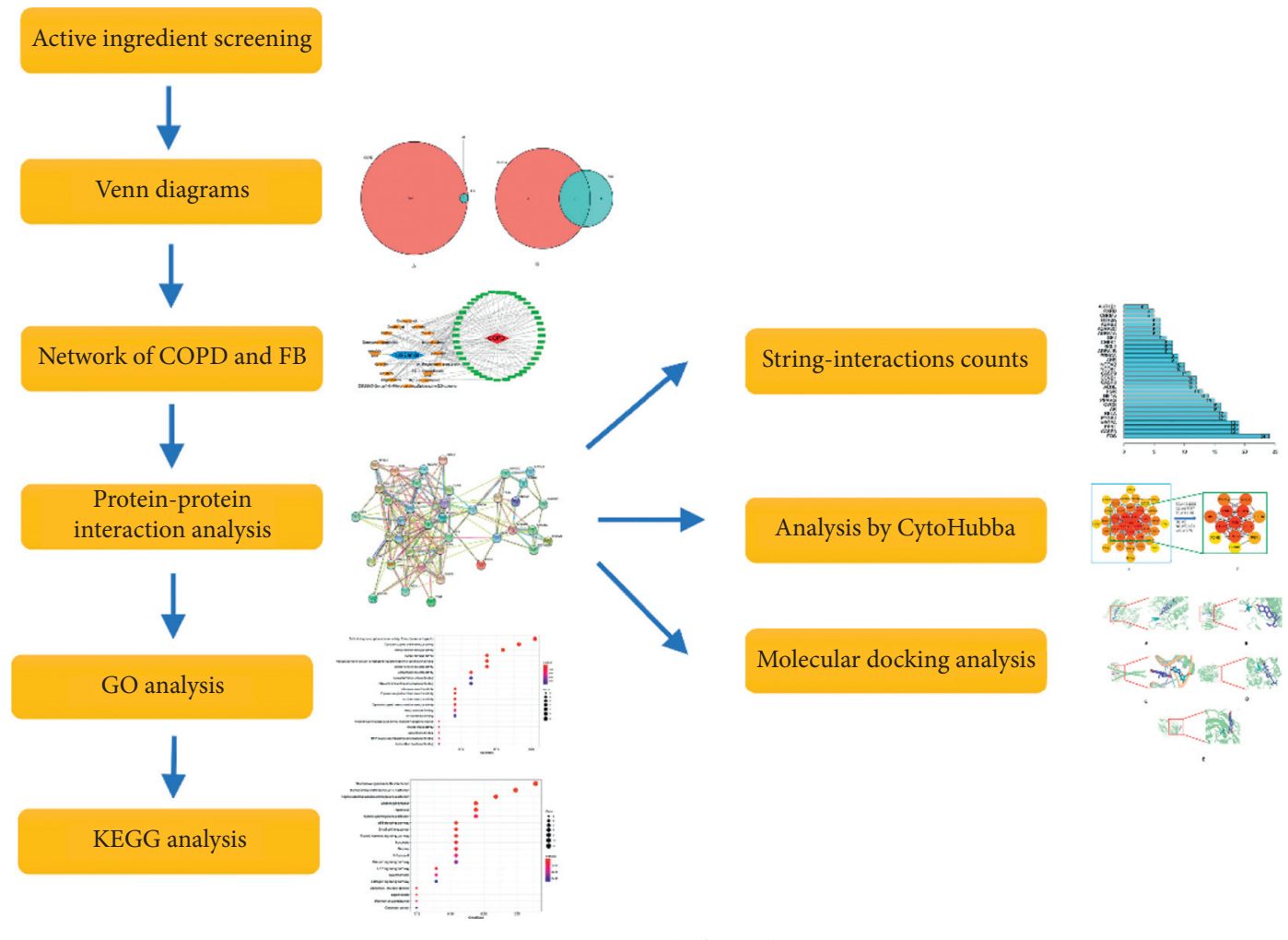

Figure 10: Article flow chart.

Epstein-Barr virus infections. COPD is an independent risk factor for non-small-cell lung cancer (NSCLC) and significantly associated with lung cancer mortality [50]. Fuzi reduced the proportion of Treg cells, decreased serum levels of cytokines and transforming growth factor (TGF)- $\beta$, and downregulated the expression of programmed death ligand-1 in mice, which suggests that Fuzi has immunomodulation properties to improve radiotherapy against lung cancer [51]. In this study, the pathways mentioned above were enhanced as well.

In the network we set up, FOS may be the core target gene. In addition, CASP 3 and VEGFA may be key target genes. The overexpression of FOS and CASP3 suggests excessive apoptosis [52]. Additionally, activated c-Fos can influence colon cancer invasion [53]; c-Fos in T24 cells can induce significant cell morphology changes, reduce viability, and increase cell death [54]. In osteosarcoma (OS), only tumors expressing both epidermal growth factor receptor (EGFR) and c-Fos respond to anti-EGFR therapy [55]. CASP3 plays a central role in executing cell apoptosis; carcinogenesis studies have found that CASP3 polymorphisms and smoking interactions were related to a higher risk of lung cancer [56], whereas lower expression of CASP3 is linked to a higher risk of NSCLC $[57,58]$. Although no difference in the expression of ESR1 was observed between patients and healthy individuals, the increased expression of enzymes involved in the local synthesis of active estrogens were observed [59]. Vascular endothelial-derived growth factor (VEGF) may play a key role in ongoing vascular remodeling processes, a key characteristic sign of COPD, in the distal lung compartments [60].

The mechanisms of action and molecular targets of FB against COPD were explored using a network pharmacology in this study. FB regulates some biological processes, such as gene expression, apoptotic processes, neuroactive ligand-receptor interaction and p53 class mediators, and cholinergic synapse, and the enhanced pathways include the IL-17 signaling and thyroid hormone signaling pathway. FB may regulate immunological function through some specific viral pathways, such as Kaposi sarcoma-associated herpesvirus infection, human immunodeficiency virus 1 infection, human cytomegalovirus infection, hepatitis $C$ and influenza $A$, and Epstein-Barr virus infection, which are associated with COPD. As judged by DC, the top 3 key genes are FOS, CASP3, and ESR1 in the PPI of FB for the treatment of COPD. Network pharmacology seems to be a suitable approach for the study of complex TCM incompatible drugs; however, it may filter compounds that may be associated with unknown target genes or lower $\mathrm{OB}$ and DL, such as BAC, BHA, and BMA identified in this study. Moreover, various formulas with effective compositions may be produced using the decocting process, instead of the original preparation methods; the compounds so obtained may have different molecular structures because they are obtained by boiling. As TCM research is difficult, although not an ideal approach, network pharmacology does offer a new and reliable approach to analyze TCMs and associate them with diseases. It can be used to 
verify whether a theory of TCM is valid through modern experimental methods in order to conveniently and accurately explain how drugs function.

\section{Data Availability}

The data used to support the findings of this study are included within the supplementary material files.

\section{Conflicts of Interest}

The authors declare that the research was conducted in the absence of any commercial or financial relationships that could be construed as potential conflicts of interest.

\section{Authors' Contributions}

$\mathrm{KN}$ conducted the main analysis and drafted this manuscript. $\mathrm{KN}$ and $\mathrm{XC}$ designed the research. YC, LZ, and ZW joined in the discussion and offered interesting ideas. $\mathrm{RC}$ and SZ assisted in the preparation of the research. ZW reviewed the text and guided the structure of the manuscript. All authors took part in the research and wrote, read, and approved the manuscript.

\section{Acknowledgments}

The authors would like to thank Editage (http://www. editage.com) for English language editing and biowolf (http://www.biowolf.cn/) for guidance on Internet pharmacology methods and $\mathrm{R}$ language. This work was supported by the National Key R\&D Program of China (2018YFC2002500).

\section{Supplementary Materials}

Supplementary Table 1. Gene targets of Fuzi. Supplementary Table 2. Gene targets of Banxia. Supplementary Table 3. Number of targets corresponding to different components. Supplementary Table 4. The data of GO enrichment analysis. Supplementary Table 5. The data of KEGG enrichment analysis. (Supplementary Materials)

\section{References}

[1] L. Tanner and A. B. Single, "Animal models reflecting chronic obstructive pulmonary disease and related respiratory disorders: translating pre-clinical data into clinical relevance," Journal of Innate Immunity, vol. 12, no. 3, pp. 203-225, 2019.

[2] Y.-N. Liao, W.-L. Hu, H.-J. Chen, and Y.-C. Hung, "The use of Chinese herbal medicine in the treatment of chronic obstructive pulmonary disease (COPD)," The American Journal of Chinese Medicine, vol. 45, no. 2, pp. 225-238, 2017.

[3] P. Zhao, J. Li, Y. Tian et al., "Restoring Th17/Treg balance via modulation of STAT3 and STAT5 activation contributes to the amelioration of chronic obstructive pulmonary disease by Bufei Yishen formula," Journal of Ethnopharmacology, vol. 217, pp. 152-162, 2018.

[4] W. Du, J. Su, D. Ye, Y. Wang, Q. Huang, and X. Gong, "Pinellia ternata attenuates mucus secretion and airway inflammation after inhaled corticosteroid withdrawal in COPD rats," The American Journal of Chinese Medicine, vol. 44, no. 5, pp. 1027-1041, 2016.

[5] S. T. Gulen, U. Eryilmaz, M. Yilmaz, and F. Karadag, "Left ventricular dysfunction in relation with systemic inflammation in chronic obstructive pulmonary disease patients," The Korean Journal of Internal Medicine, vol. 34, no. 3, pp. 569578, 2019.

[6] G. Zhou, L. Tang, X. Zhou, T. Wang, Z. Kou, and Z. Wang, "A review on phytochemistry and pharmacological activities of the processed lateral root of aconitum carmichaelii Debeaux," Journal of Ethnopharmacology, vol. 160, pp. 173-193, 2015.

[7] N. Li, L. L. Li, C. X. Li, Y. H. Chen, and X. L. Li, "Analysis of the historical evolution of "eighteen incompatible medicaments" and "nineteen medicaments of mutual restraint" of TCM and its clinical application," China Pharmacy, vol. 30, pp. 513-517, 2019, in Chinese.

[8] J. Wu, Z. Cheng, L. Zhu et al., "Coadministration of Pinellia ternata can significantly reduce Aconitum carmichaelii to inhibit CYP3A activity in rats," Evidence-based Complementary and Alternative Medicine, eCAM, vol. 2014, Article ID 734867, 10 pages, 2014.

[9] X. W. Mo, W. X. He, X. H. Liu, J. Zhang, and H. Deng, "Study on the safety of compatibility of aconite and Pinellia ternata in the treatment of malignant tumors," Psychologist, vol. 24, no. 27, pp. 127-129, 2018, in Chinese.

[10] T. Yu, S. Xiao, F. Cao, and L. M. Liu, "Study on the compatibility of aconite and pinellia ternate in "shanghan zabing Lun'” Journal of Basic Chinese Medicine, vol. 23, no. 11, pp. 626-627, 2017, in Chinese.

[11] J. Y. Li, "To observe the curative effect of Xiaoqinglong-Jiafuzi-decoction on chronic obstructive pulmonary disease," Medical Diet and Health, vol. 18, no. 12, p. 11, 2020, in Chinese.

[12] D. F. Chen, Z. L. Zhang, H. B. Xiao, and L. H. Xiao, “Analysis of clinical effect of "Five-step method of differentiating yang deficiency" combined with "Xiaoqinglong decoction plus aconite" in the treatment of allergic rhinitis," China Practical Medical, vol. 12, no. 14, pp. 128-129, 2017, in Chinese.

[13] H. Liu, L. Zeng, K. Yang, and G. Zhang, "A network pharmacology approach to explore the pharmacological mechanism of Xiaoyao powder on anovulatory infertility," EvidenceBased Complementary and Alternative Medicine, vol. 2016, Article ID 2960372, 13 pages, 2016.

[14] Y. Chen, J. Wei, Y. Zhang et al., "Anti-endometriosis mechanism of jiawei foshou san based on network pharmacology," Frontiers in Pharmacology, vol. 9, p. 811, 2018.

[15] S. J. Yue, J. Liu, W. W. Feng et al., "System pharmacologybased dissection of the synergistic mechanism of Huangqi and huanglian for diabetes mellitus," Frontiers in Pharmacology, vol. 8, p. 694, 2017.

[16] X. Xing, S. Chen, L. Li et al., "The active components of Fuzheng Huayu formula and their potential mechanism of action in inhibiting the hepatic stellate cells viability-a network pharmacology and transcriptomics approach," Frontiers in Pharmacology, vol. 9, p. 525, 2018.

[17] J. Ru, P. Li, J. Wang et al., "TCMSP: a database of systems pharmacology for drug discovery from herbal medicines," Journal of Cheminformatics, vol. 6, p. 13, 2014.

[18] J. Li, P. Zhao, Y. Li, Y. Tian, and Y. Wang, "Systems pharmacology-based dissection of mechanisms of Chinese medicinal formula Bufei Yishen as an effective treatment for chronic obstructive pulmonary disease," Scientific Reports, vol. 5, Article ID 15290, 2015. 
[19] V. Law, C. Knox, Y. Djoumbou et al., "DrugBank 4.0: shedding new light on drug metabolism," Nucleic Acids Research, vol. 42, no. D1, pp. D1091-D1097, 2014.

[20] L. L. Miao, Q. M. Zhou, C. Peng, C. W. Meng, X. Y. Wang, and L. Xiong, "Aconitum carmichaelii discrimination of the geographical origin of the lateral roots of using the fingerprint, multicomponent quantification, and chemometric methods," Molecules (Basel, Switzerland), vol. 24, no. 22, 4124 pages, 2019.

[21] Y. Tang, M. Li, J. Wang, Y. Pan, and F.-X. Wu, "CytoNCA: a cytoscape plugin for centrality analysis and evaluation of protein interaction networks," Biosystems, vol. 127, pp. 67-72, 2015.

[22] C.-H. Chin, S.-H. Chen, H.-H. Wu, C.-W. Ho, M.-T. Ko, and C.-Y. Lin, "CytoHubba: identifying hub objects and subnetworks from complex interactome," BMC Systems Biology, vol. 8, no. Suppl 4, p. S11, 2014.

[23] D. W. Huang, B. T. Sherman, and R. A. Lempicki, "Systematic and integrative analysis of large gene lists using DAVID bioinformatics resources," Nature Protocols, vol. 4, no. 1, pp. 44-57, 2009.

[24] L. Gatto, L. M. Breckels, T. Naake, and S. Gibb, "Visualization of proteomics data using R and bioconductor," Proteomics, vol. 15, no. 8, pp. 1375-1389, 2015.

[25] B. Boezio, K. Audouze, P. Ducrot, and O. Taboureau, "Network-based approaches in pharmacology," Molecular Informatics, vol. 36, no. 10, 2017.

[26] K. Ohbuchi, C. Miyagi, Y. Suzuki et al., "Ignavine: a novel allosteric modulator of the $\mu$ opioid receptor," Scientific Reports, vol. 6, Article ID 31748, 2016.

[27] R. Roy, D. Pal, S. Sur, S. Mandal, P. Saha, and C. K. Panda, "Pongapin and Karanjin, furanoflavanoids of Pongamia pinnata, induce G2/M arrest and apoptosis in cervical cancer cells by differential reactive oxygen species modulation, DNA damage, and nuclear factor kappa-light-chain-enhancer of activated B cell signaling," Phytotherapy Research, vol. 33, no. 4, pp. 1084-1094, 2019.

[28] M. Bose, M. Chakraborty, S. Bhattacharya, D. Mukherjee, S. Mandal, and R. Mishra, "Prevention of arthritis markers in experimental animal and inflammation signalling in macrophage by karanjin isolated fromPongamia pinnataSeed extract," Phytotherapy Research, vol. 28, no. 8, pp. 1188-1195, 2014.

[29] L. Ye, S. Gao, Q. Feng et al., "Development and validation of a highly sensitive UPLC-MS/MS method for simultaneous determination of aconitine, mesaconitine, hypaconitine, and five of their metabolites in rat blood and its application to a pharmacokinetics study of aconitine, mesaconitine, and hypaconitine," Xenobiotica, vol. 42, no. 6, pp. 518-525, 2012.

[30] S. Saadat and J Boskabadi, M. H. Boskabady, Contribution of potassium channels, beta2-adrenergic and histamine $\mathrm{H} 1 \mathrm{re-}$ ceptors in the relaxant effect of baicalein on rat tracheal smooth muscle," Iranian Journal of Basic Medical Sciences, vol. 22, no. 11, pp. 1347-1352, 2019.

[31] C. H. Wiegman, C. Michaeloudes, G. Haji et al., "Oxidative stress-induced mitochondrial dysfunction drives inflammation and airway smooth muscle remodeling in patients with chronic obstructive pulmonary disease," Journal of Allergy and Clinical Immunology, vol. 136, no. 3, pp. 769-780, 2015.

[32] C. L. Li, Q. S, Y. Y et al., "Recuperating lung decoction attenuates the oxidative stress state of chronic obstructive pulmonary disease by inhibiting the MAPK/AP-1 signal pathway and regulating $-\gamma$ GCS," Evidence-Based
Complementary and Alternative Medicine, vol. 2017, Article ID 9264914, 12 pages, 2017.

[33] D. Zong, J. Li, S. Cai et al., "Notch1 regulates endothelial apoptosis via the ERK pathway in chronic obstructive pulmonary disease," American Journal of Physiology-Cell Physiology, vol. 315, no. 3, pp. C330-C340, 2018.

[34] M. Vaz, S. Rajasekaran, H. R. Potteti, and S. P. Reddy, "Myeloid-specific Fos-related antigen-1 regulates cigarette smoke-induced lung inflammation, not emphysema, in mice," American Journal of Respiratory Cell and Molecular Biology, vol. 53, no. 1, pp. 125-134, 2015.

[35] A. Birnhuber, V. Biasin, D. Schnoegl, L. M. Marsh, and G. Kwapiszewska, "Transcription factor Fra-2 and its emerging role in matrix deposition, proliferation and inflammation in chronic lung diseases," Cellular Signalling, vol. 64, Article ID 109408, 2019.

[36] Y.-P. Wu, Y.-F. Wu, C. Zhang et al., "Activating transcription factor 3 Is essential for cigarette smoke-induced mucin expression via interaction with activator protein-1," The American Journal of Pathology, vol. 187, no. 2, pp. 280-291, 2017.

[37] H.-H. Hou, H.-C. Wang, S.-L. Cheng, Y.-F. Chen, K.-Z. Lu, and C.-J. Yu, "MMP-12 activates protease-activated receptor1 , upregulates placenta growth factor, and leads to pulmonary emphysema," American Journal of Physiology-Lung Cellular and Molecular Physiology, vol. 315, no. 3, pp. L432-L442, 2018.

[38] K. Mizumura, S. Maruoka, T. Shimizu, and Y. Gon, "Autophagy, selective autophagy, and necroptosis in COPD," International Journal of Chronic Obstructive Pulmonary Disease, vol. 13, pp. 3165-3172, 2018.

[39] M. S. Eapen, P. M. Hansbro, K. McAlinden et al., "Abnormal M1/M2 macrophage phenotype profiles in the small airway wall and lumen in smokers and chronic obstructive pulmonary disease (COPD)," Scientific Reports, vol. 7, no. 1, p. 13392, 2017.

[40] J. Knobloch, D. Jungck, J. Kronsbein, E. Stoelben, K. Ito, and A. Koch, "LABAs and p38MAPK inhibitors reverse the corticosteroid-insensitivity of IL-8 in airway smooth muscle cells of COPD," Journal of Clinical Medicine, vol. 8, no. 12, 2058 pages, 2019.

[41] G. Hodge, E. Roscioli, H. Jersmann et al., "Steroid resistance in COPD is associated with impaired molecular chaperone Hsp90 expression by pro-inflammatory lymphocytes," Respiratory Research, vol. 17, no. 1, p. 135, 2016.

[42] M. M. Gouda, S. B. Shaikh, D. Chengappa, I. Kandhal, A. Shetty, and Y. Bhandary, "Changes in the expression level of IL-17A and p53-fibrinolytic system in smokers with or without COPD," Molecular Biology Reports, vol. 45, no. 6, pp. 2835-2841, 2018.

[43] G. Anzalone, G. D. Albano, A. M. Montalbano et al., "IL-17Aassociated IKK-alpha signalling induced TSLP production in epithelial cells of COPD patients," Experimental \& Molecular Medicine, vol. 50, no. 10, p. 131, 2018.

[44] M. Niwa, T. Fujisawa, K. Mori et al., "IL-17A attenuates IFN- $\lambda$ expression by inducing suppressor of cytokine signaling expression in airway epithelium," The Journal of Immunology, vol. 201, no. 8, pp. 2392-2402, 2018.

[45] C. Gu, Y. Li, J. Liu et al., "LncRNA-mediated SIRT1/FoxO3a and SIRT1/p53 signaling pathways regulate type II alveolar epithelial cell senescence in patients with chronic obstructive pulmonary disease," Molecular Medicine Reports, vol. 15, no. 5, pp. 3129-3134, 2017. 
[46] T. Ahmad, I. K. Sundar, C. A. Lerner et al., "Impaired mitophagy leads to cigarette smoke stress-induced cellular senescence: implications for chronic obstructive pulmonary disease," The FASEB Journal, vol. 29, no. 7, pp. 2912-2929, 2015.

[47] X.-F. Zhang, S.-Y. Xiang, W.-Y. Geng et al., "Electro-acupuncture regulates the cholinergic anti-inflammatory pathway in a rat model of chronic obstructive pulmonary disease," Journal of Integrative Medicine, vol. 16, no. 6, pp. 418-426, 2018.

[48] J. Milara, A. Cervera, A. De Diego et al., "Non-neuronal cholinergic system contributes to corticosteroid resistance in chronic obstructive pulmonary disease patients," Respiratory Research, vol. 17, no. 1, p. 145, 2016.

[49] R. Hewitt, H. Farne, A. Ritchie, E. Luke, S. L. Johnston, and P. Mallia, "The role of viral infections in exacerbations of chronic obstructive pulmonary disease and asthma," Therapeutic Advances in Respiratory Disease, vol. 10, no. 2, pp. 158-174, 2016.

[50] E. Ytterstad, P. Moe, and A. Hjalmarsen, "COPD in primary lung cancer patients: prevalence and mortality," International Journal of Chronic Obstructive Pulmonary Disease, vol. 11, pp. 625-636, 2016.

[51] Q. Zhang, X. Chen, Y. Luo, H. Ren, and T. Qiao, "Fuzi enhances anti-tumor efficacy of radiotherapy on lung cancer," Journal of Cancer, vol. 8, no. 19, pp. 3945-3951, 2017.

[52] L. Wen, L. Liu, L. Tong et al., "NDRG4 prevents cerebral ischemia/reperfusion injury by inhibiting neuronal apoptosis," Genes é Diseases, vol. 6, no. 4, pp. 448-454, 2019.

[53] Y. Ding, K. Hao, Z. Li et al., "c-Fos separation from Lamin A/ $\mathrm{C}$ by GDF15 promotes colon cancer invasion and metastasis in inflammatory microenvironment," Journal of Cellular Physiology, vol. 235, no. 5, pp. 4407-4421, 2020.

[54] T. Zhang, J. He, S. Zhang et al., "Brazilin induces T24 cell death through c-Fos and GADD $45 \beta$ independently regulated genes and pathways," IUBMB Life, vol. 70, no. 11, pp. 1101-1110, 2018.

[55] M. Linder, E. Glitzner, S. Srivatsa et al., "EGFR is required for FOS-dependent bone tumour development via RSK2/CREB signalling," EMBO Molecular Medicine, vol. 10, no. 11, 2018.

[56] J. Lin, Y. Zhang, H. Wang et al., "Genetic polymorphisms in the apoptosis-associated gene CASP3 and the risk of lung cancer in Chinese population," PloS One, vol. 11, no. 10, Article ID e0164358, 2016.

[57] R. Cui, T. Kim, M. Fassan et al., "MicroRNA-224 is implicated in lung cancer pathogenesis through targeting caspase- 3 and caspase-7," Oncotarget, vol. 6, no. 26, pp. 21802-21815, 2015.

[58] J. Javid, R. Mir, and A. Saxena, "Involvement of CASP3 promoter polymorphism $(-1337 \mathrm{C}>\mathrm{G})$ in the development and progression of non-small cell lung cancer," Tumor Biology, vol. 37, no. 7, pp. 9255-9262, 2016.

[59] G. F. J. Konings, N. L. Reynaert, B. Delvoux et al., "Increased levels of enzymes involved in local estradiol synthesis in chronic obstructive pulmonary disease," Molecular and Cellular Endocrinology, vol. 443, pp. 23-31, 2017.

[60] G. Westergren-Thorsson, M. Bagher, A. Andersson-Sjöland et al., "VEGF synthesis is induced by prostacyclin and TGF- $\beta$ in distal lung fibroblasts from COPD patients and control subjects: implications for pulmonary vascular remodelling," Respirology, vol. 23, no. 1, pp. 68-75, 2018. 